Revue des patrimoines

27 | 2015

Le cheval et ses patrimoines (2e partie)

\title{
Catégories équines : la race et le type
}

\section{Amandine Souvré}

\section{OpenEdition}

Journals

Édition électronique

URL : http://journals.openedition.org/insitu/12183

DOI : 10.4000/insitu.12183

ISSN : 1630-7305

\section{Éditeur}

Ministère de la culture

\section{Référence électronique}

Amandine Souvré, "Catégories équines : la race et le type », In Situ [En ligne], 27 | 2015, mis en ligne le 02 novembre 2015, consulté le 03 mai 2019. URL : http://journals.openedition.org/insitu/12183 ; DOI : 10.4000/insitu. 12183

Ce document a été généré automatiquement le 3 mai 2019.

\section{(2) $\oplus \Theta \Theta$}

In Situ Revues des patrimoines est mis à disposition selon les termes de la licence Creative Commons Attribution - Pas d'Utilisation Commerciale - Pas de Modification 4.0 International. 


\title{
Catégories équines : la race et le type
}

\author{
Amandine Souvré
}

1 Le présent article trouve son origine dans les recherches effectuées pour la thèse d'École des chartes intitulée "L'œuvre des Haras, entre amélioration animale et création architecturale: l'implantation du dépôt d'étalons de Cluny (premier quart du xIx siècle) », dirigée par M. Jean-Michel Leniaud et soutenue le 11 février 2014 devant un jury composé de MM. Bernard Denis, Jean-Pierre Digard et Daniel Roche ${ }^{1}$. La communication dont il est issu aspirait, à partir des éléments particuliers rencontrés dans ce cadre précis, à développer un propos plus général sur les races équines - le but étant moins d'avancer des assertions que d'interroger ce concept complexe, du point de vue de l'historien.

\section{La notion de race : une élaboration progressive}

2 La notion de race, telle qu'elle est entendue aujourd'hui, est le fruit d'une construction progressive. Parvenir à la définition actuelle, désignant un "groupe d'individus morphologiquement homogène, aux caractéristiques héréditairement transmissibles, adaptés aux conditions du milieu tant humain que naturel $»^{2}$, a nécessité une lente maturation et l'élaboration d'outils parmi lesquels figurent au premier chef les livres généalogiques et les standards, ces critères permettant de déterminer les individus à inclure ou exclure dans une race. L'existence de livres généalogiques témoigne d'une prise en compte et d'une certaine maîtrise, pragmatique tout au moins, de l'hérédité, sans lesquelles nulle politique d'élevage n'est envisageable.

3 Or, aucun de ces instruments n'existait à l'époque de la création des Haras impériaux. Si le stud-book, livre généalogique spécialement dévolu à l'espèce équine, a vu le jour en Angleterre dès 1791, il fallut attendre 1833 pour qu'une ordonnance royale instaurât une mesure similaire en France, avec l'ouverture d'un «registre-matricule» destiné à enregistrer les chevaux de « race pure $»^{3}$. Et ce n'est que dans le dernier quart du siècle que cette première initiative restée plusieurs décennies isolée fut suivie et complétée : 
ouverture du livre généalogique du cheval nivernais en 1880, du percheron en 1883, du boulonnais en 1885 , etc. ${ }^{4}$.

Il ne faudrait pas croire cependant que l'on a attendu l'outil pour posséder le concept : le terme de race, employé indifféremment de celui d'“ espèce ", préexistait aux stud-books et était omniprésent dans le discours bien avant que l'on n'échafaudât une notion précisément circonscrite grâce aux standards. C'est que se perpétuaient déjà, localement ou régionalement, des populations sinon homogènes du moins présentant des caractères communs : il s'agit des « races-terroir $»^{5}$, produits d'un milieu et d'une société donnés, qui portent d'ailleurs le plus souvent le nom de leur aire géographique. Malheureusement, de nombreux écrans se dressent entre ces races anciennes et l'observateur actuel, qui l'empêchent de concevoir pleinement les réalités auxquelles renvoie la plume d'un homme du début $\mathrm{du}$ xix siècle lorsqu'il évoque un étalon normand, une jument charolaise ou un cheval des Dombes.

\section{La race et l'historien}

Figure 1

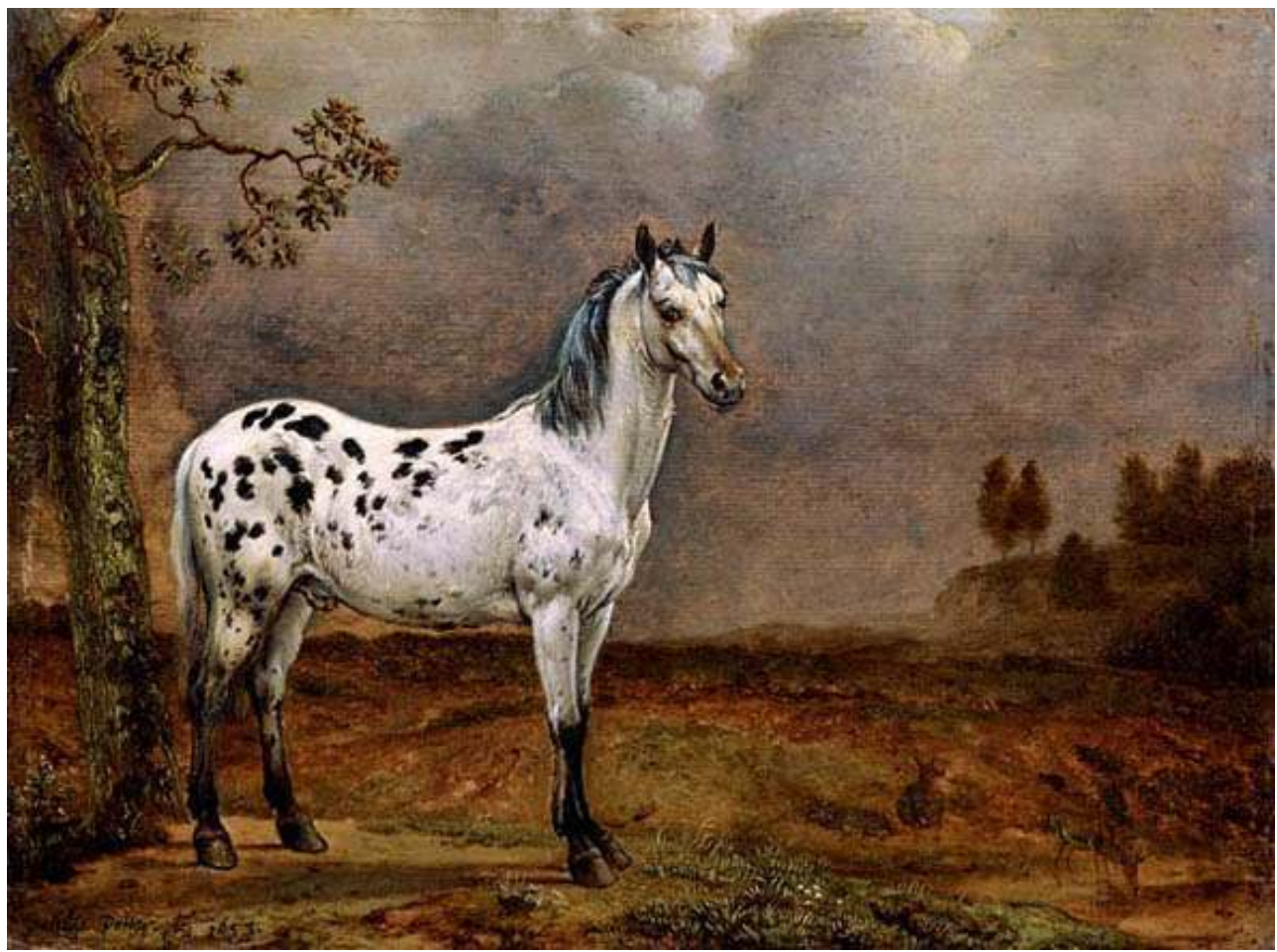

Le cheval pie, Paulus Potter, musée du Louvre, 1653.

Phot. Schormans, Jean. (C) Réunion des musées nationaux. Base Joconde : 000PE008543. 
Figure 2

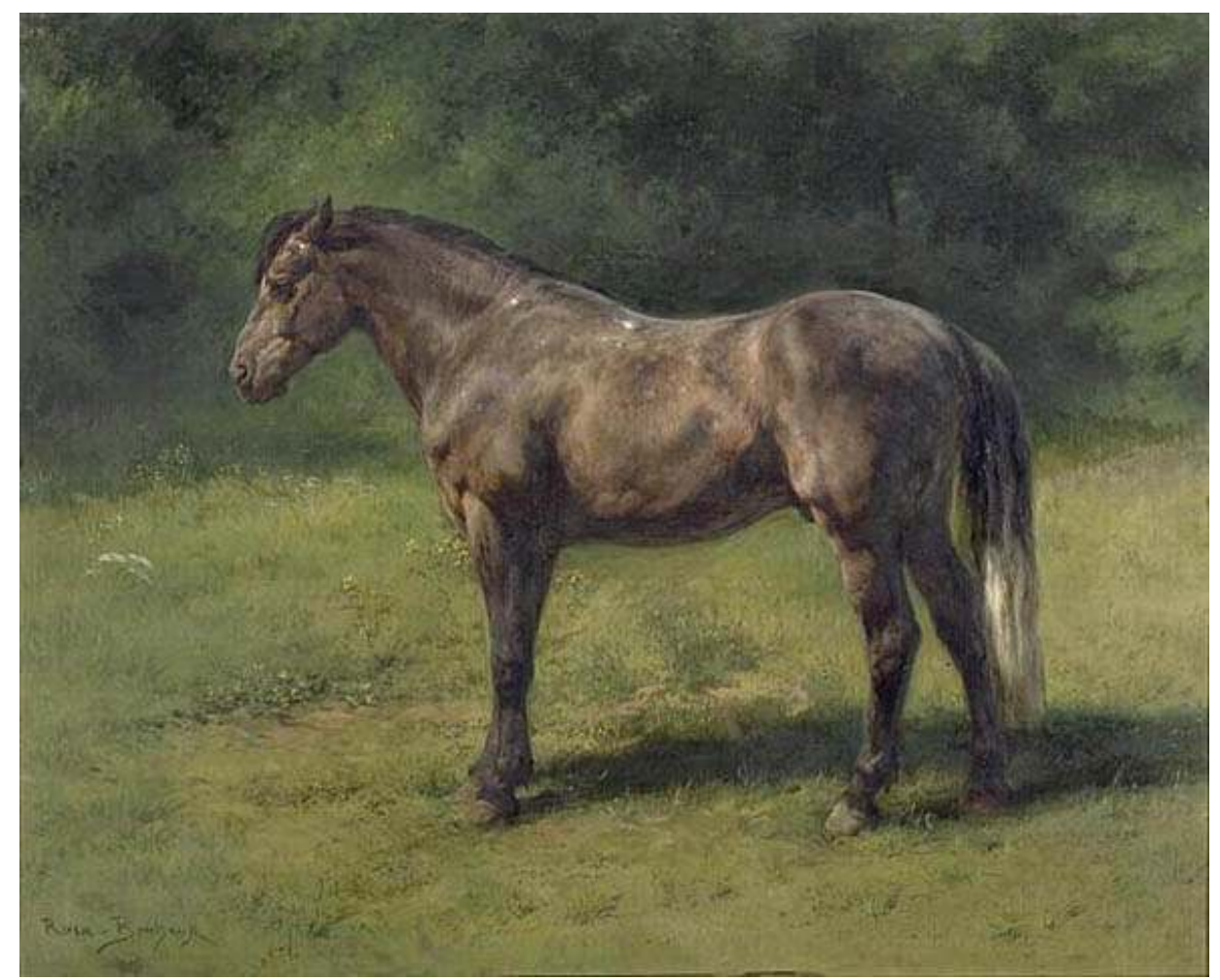

Étude de cheval gris au vert, Rosa Bonheur, musée national du château de Fontainebleau, deuxième moitié du XIXe siècle.

Phot. Lagiewski, Jean-Pierre. (c) Réunion des musées nationaux. Base Joconde : 50130000054

Le premier obstacle auquel se trouve confronté l'historien réside dans l'absence de représentation réaliste de la diversité des races sous le Premier Empire. Même s'il est bien entendu qu'une image fixe en deux dimensions est un moyen fort imparfait de connaître la morphologie d'un animal, c'est un recours commode qui nous a fait défaut: le formidable instrument documentaire que constitue la photographie, en vertu de sa relative objectivité, fut précisément inventé dans cette contrée mais à une date trop tardive pour nous avoir été d'aucune utilité dans ce travail. Quant à la peinture, elle ne s'occupait plus ou pas encore de réalisme : en dehors de toutes considérations artistiques, on ne peut que regretter que le premier quart du XIX ${ }^{e}$ siècle n'ait pas connu un Paulus Potter (fig. 1) ou une Rosa Bonheur (fig. 2) pour pallier le manque de véracité inhérent au style d'un peintre tel que David (fig. 3). Il nous faut toutefois rendre justice - tout en gardant à l'esprit qu'ils ne concernent que des favoris de prince et n'apporteront jamais un quelconque enseignement sur le cheval de selle du Morvan, par exemple - aux portraits de chevaux réalisés pour l'Empereur ; s'ils ne sont pas exempts de conventions, ils traduisent malgré tout des individualités à la variété authentique (fig. 4). 
Figure 3

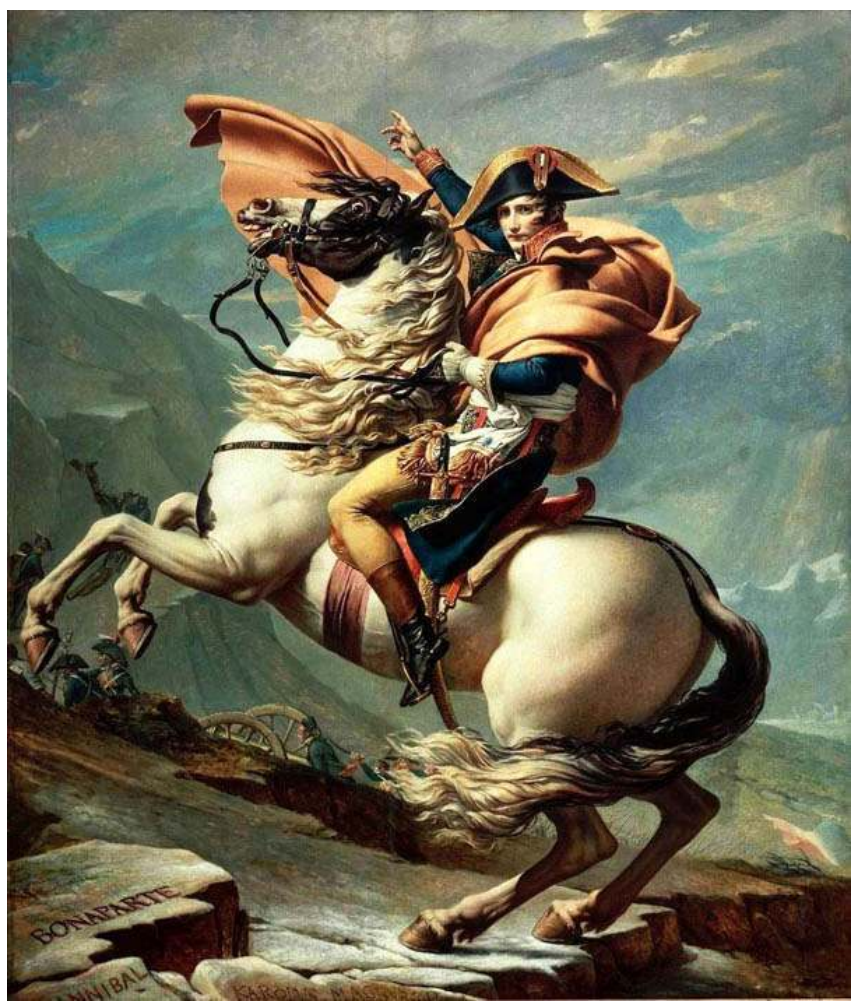

Le premier Consul franchissant les Alpes au col du Grand Saint-Bernard, Jacques Louis David, musée national des châteaux de Malmaison et de Bois-Préau, 1800.

Phot. Blot, Gérard. (c) Réunion des musées nationaux. Base Joconde : 00000095069. 
Figure 4

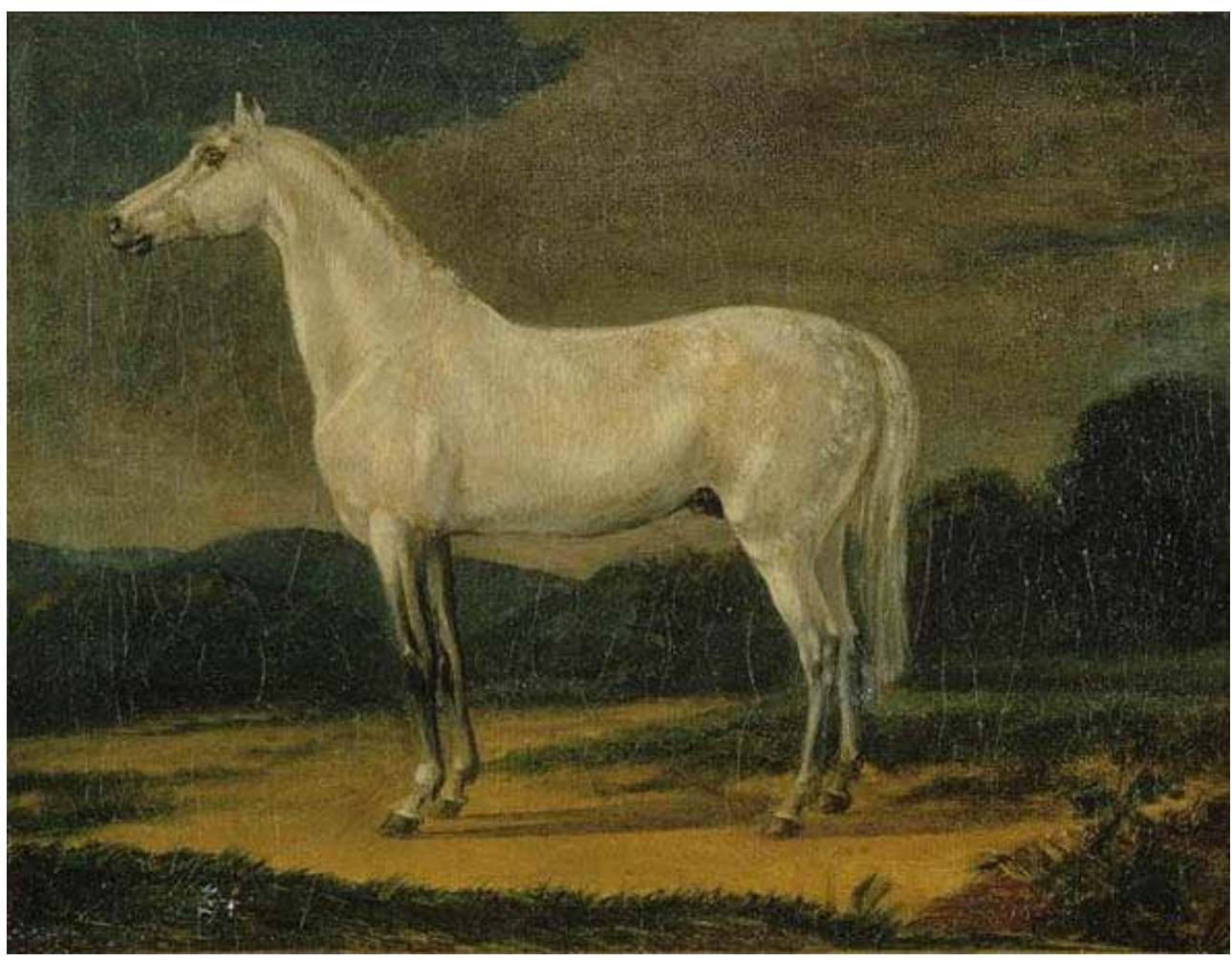

Cheval de Napoléon ler : Le Vizir, Pierre Martinet, musée national des châteaux de Malmaison et de Bois-Préau, 1806.

Phot. Mathéus. @ Réunion des musées nationaux. Base Joconde : 50160000014.

Les sources visuelles ne nous étant d'aucun secours documentaire, c'est à la seule force des mots qu'il a fallu s'en remettre pour faire surgir du papier l'image de l'animal. Mais les sources écrites ne sont pas davantage dépourvues d'écueils et opposent au chercheur leur lot de difficultés. Tout d'abord, le vocabulaire destiné à sa description a beau être extraordinairement riche, l'exercice a beau être très ancien et fréquent, il est impossible de donner une idée fidèle et suffisante du cheval par le langage. Pis, il est extrêmement difficile de se départir d'une subjectivité plus ou moins envahissante. Dans le cas du dépôt d'étalons de Cluny, il faut notamment tenir compte du fait que la source principale émane des inspecteurs généraux des Haras, fins connaisseurs certes, mais aussi agents centraux de l'État, imprégnés par conséquent des conceptions et préjugés de la classe dirigeante : l'administration nous instruit sur le « cheval de l'administration » .

7 Aux limites imposées par la subjectivité s'ajoute la fréquente indétermination dans laquelle se trouvaient les contemporains pour décrire les différentes races qu'ils étaient amenés à observer. Nombreux sont ceux, d'un terme à l'autre de la période, qui ont avoué l'incapacité où ils étaient de caractériser les chevaux s'offrant à leur regard :

Les juments de la vallée de Cluny, de celle de la Saône et de quelques autres rivières sont plus fortes que celles du Charolais et du Morvan. Elles n'appartiennent à aucune race distincte, viennent de plusieurs cantons de France ${ }^{7}$.

Les espèces de juments que [le dépôt d'étalons de Cluny] a à servir sont les juments bressandes, bourguignonnes, morvandaises, charolaises. Ces races sont abâtardies, se confondent et sont peu prononcées ${ }^{8}$. 
Ces hésitations constituent une véritable impasse : quand le témoin direct n'a pas su trancher, que peut l'historien ? La dernière citation pointe, en outre, une ultime entrave à notre connaissance des races équines du début du xix siècle : l'idée de dégénérescence, notion héritée de Buffon, a fait communément admettre que toutes les races s'étaient abâtardies 9 . Ce préjugé largement partagé (et pour lequel il est difficile de déterminer la part de mythe et de réelle observation) fait que l'on abandonnait bien vite toute velléité de description précise pour se retrancher derrière ce qualificatif commode, privant par là la postérité de précieuses indications.

\section{Vers une meilleure connaissance}

En dépit de toutes ces réserves, force est de reconnaitre la possibilité, mise en évidence par le constat d'un consensus, de faire progresser notre connaissance des races: les sources parlent unanimement de chevaux limousins, normands, arabes, anglais, danois, suisses, espagnols, etc. Il ne paraît pas abusif de partir du postulat que ces dénominations n'étaient pas attribuées au hasard mais renvoyaient au contraire, dans l'esprit des contemporains, à des différenciations valables. Partant, il nous incombe de chercher à mieux cerner ces représentations abolies. Les archives du dépôt d'étalons de Cluny (ainsi que de tous les autres établissements des Haras, ce qui laisse un vrai travail à mener) constituent pour ce faire une ressource inestimable.

Figure 5

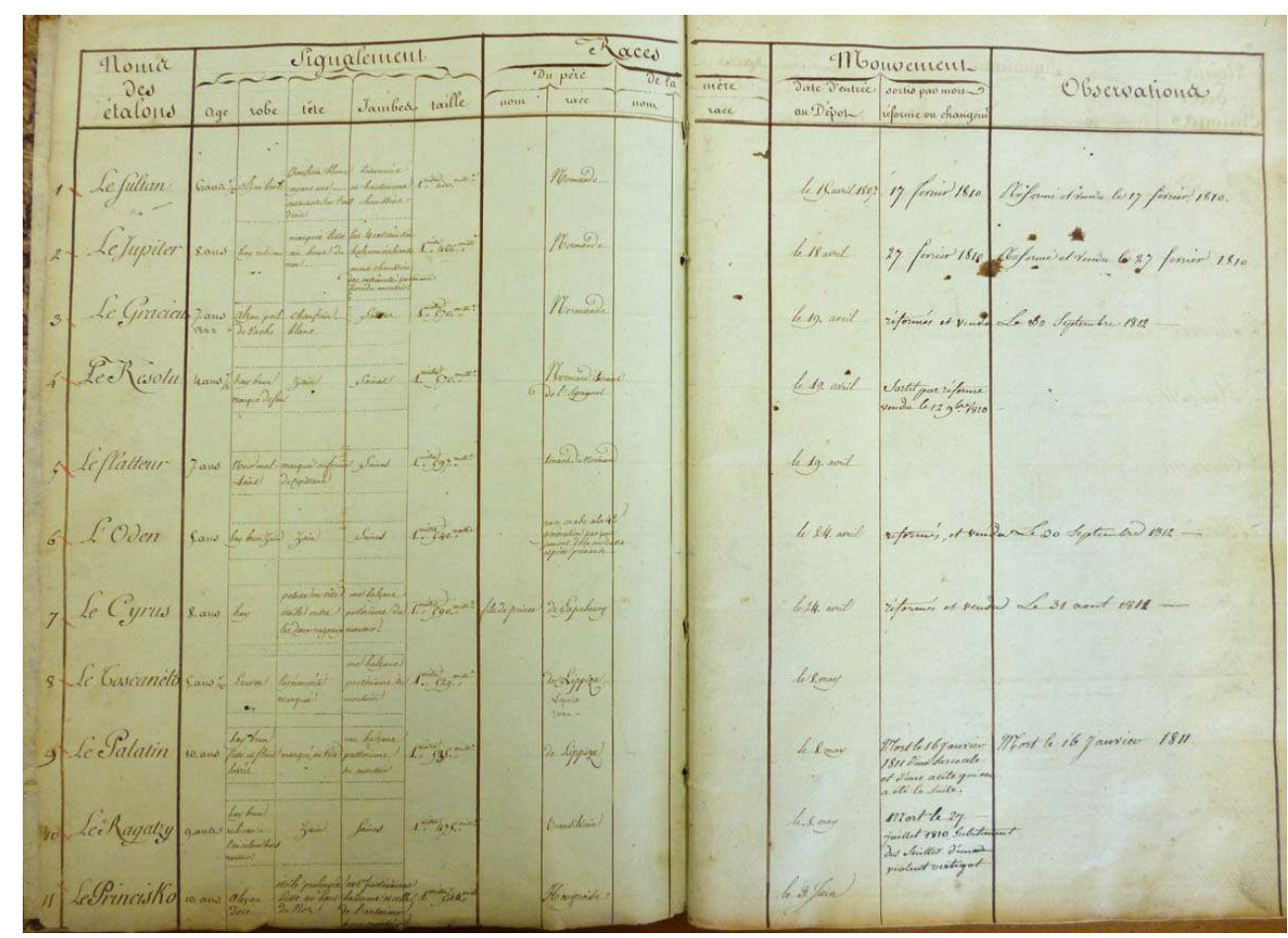

Premier registre-matricule des étalons du dépôt d'étalons de Cluny, 1807-1820.

Phot. Souvré, Amandine. (c) Archives départementales de Saône-et-Loire.

Deux natures de documents ont été particulièrement utiles pour apprécier la population chevaline vouée à la reproduction dans la circonscription du dépôt de Cluny: les registres-matricules et les registres de monte. Les premiers recensent, d'abord de 
manière très concise (fig. 5) puis de plus en plus précise (fig. 6), l'identité de chaque étalon passé au haras. Les seconds, répertoriant chaque saillie, sont d'une importance capitale puisqu'ils représentent à la fois le témoignage le plus exact de l'activité du dépôt d'étalons et l'une des rares sources permettant de se faire une idée de la jumenterie desservie, grâce au signalement succinct des poulinières. Bien qu'ils ne reflètent l'œuvre que d'un seul établissement sur la trentaine existante au début du XIX ${ }^{e}$ siècle, ces registres sont loin d'être négligeables : eu égard à la rapide extension de la circonscription (fig. 7), ils embrassent une portion de territoire et un échantillon d'équidés assez vastes pour être représentatifs. De 1807 à 1822, les écuries de Cluny virent ainsi passer 121 étalons qui saillirent en l'espace des quatre années analysées $(1807,1808,1809$ et 1822) 2025 juments dans huit départements (Saône-et-Loire, Ain, Côte-d'Or, Rhône, Loire, Allier, Nièvre, Yonne).

Figure 6

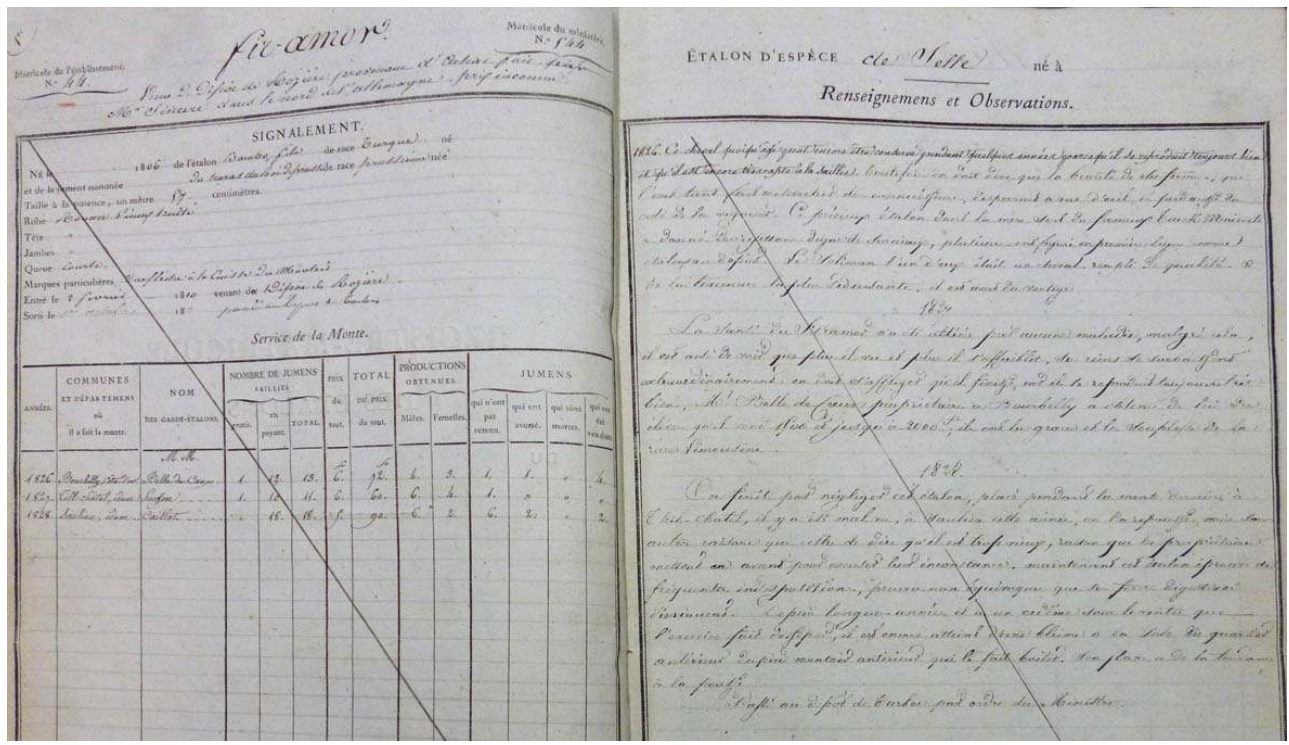

Double-page du registre-matricule consacrée à l'étalon Fir-Amor.

Phot. Souvré, Amandine. (c) Archives départementales de Saône-et-Loire. 


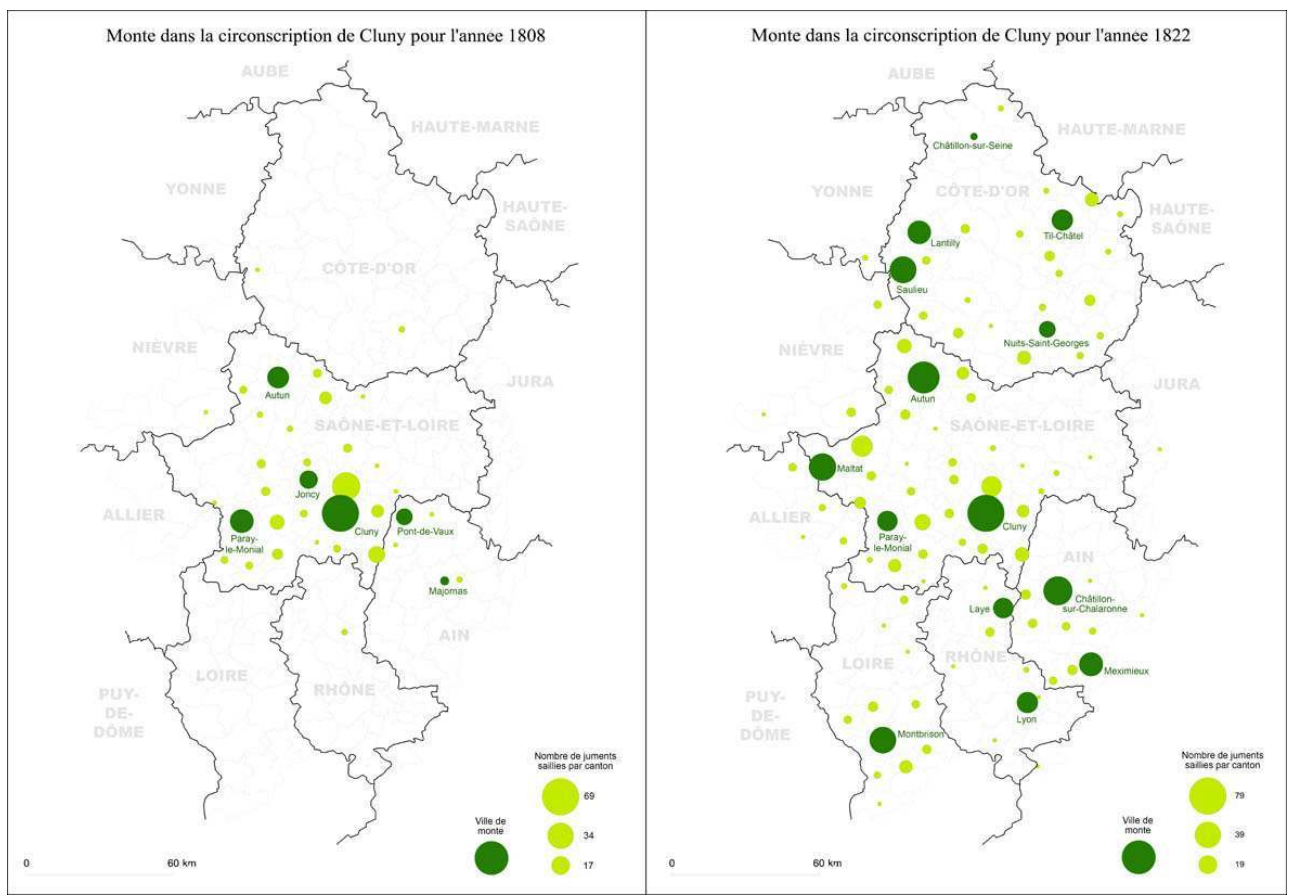

Extension territoriale de l'influence du dépôt d'étalons de Cluny entre les saisons de monte des années 1808 et 1822.

Repro. Souvré, Amandine. (c) Amandine Souvré.

Plusieurs tendances peuvent être observées concernant les races des étalons. On observe premièrement une prédominance des étalons normands, en dépit de la théorie d'élevage qui voulait que l'on améliorât l'espèce chevaline grâce à des croisements perpétuels avec du sang étranger. Les limousins étaient également bien représentés. Ce fait situe sans ambiguïté Cluny dans la hiérarchie des établissements : tandis que les haras et dépôts de premier plan recevaient des étalons étrangers pour perfectionner une population déjà "qualiteuse », on jugeait qu'il fallait, ici, en passer par une amélioration préalable au moyen des chevaux français. Les sangs anglais et arabe, les plus appréciés, n'étaient cependant pas absents et se présentaient dans des proportions comparables. Il s'agit là d'une seconde tendance digne d'attention: l'observation des dates de présence au dépôt de ces étalons étrangers atteste l'inexactitude d'une dichotomie généralisée entre un Empire anglophobe et une Restauration anglomane. Enfin, la troisième tendance est au déclin des races ibériques, très affectionnées dans les temps modernes, encore bien représentées sous l'Empire, mais marquant le pas à partir de la Restauration.

Quant aux races de juments, le premier constat à faire est celui d'une bien plus grande diversité. Les races présentes chez les étalons, qu'elles soient françaises ou étrangères (normande, limousine, arabe, anglaise, espagnole, napolitaine, turque, hongroise, hanovrienne, danoise, polonaise, comtoise, suisse, bretonne, cauchoise, etc.), se retrouvent aux côtés de nombreuses autres, plus spécifiquement françaises mais pas seulement: morvandelle, bressane, charolaise, bourbonnaise, nivernaise, auvergnate, ardennaise, camargue, savoyarde, poitevine, corse, sarde, portugaise, tartare, persane, autrichienne, russe, caucasienne et même éthiopienne (fig. 8). Le deuxième élément à souligner concerne les nombreuses appellations désignant les juments comme «communes» ou «du pays» (dans des "pays» aussi différents que les environs de 
Montbrison, de Bourbon-Lancy ou de Lantilly). Ces qualificatifs, conjugués à la structure de la propriété des poulinières, indiquent un élevage à deux facettes : d'une part, une poignée d'éleveurs étaient capables de conduire à la saillie plusieurs juments de race étrangère (jusqu'à deux dizaines pour un même homme), d'autre part, la grande majorité des éleveurs était en réalité constituée de petits propriétaires menant une seule jument locale à l'étalon, dans un but que l'on suppose modeste (renouvellement de la force de travail ou faible rémunération d'appoint).

Figure 8

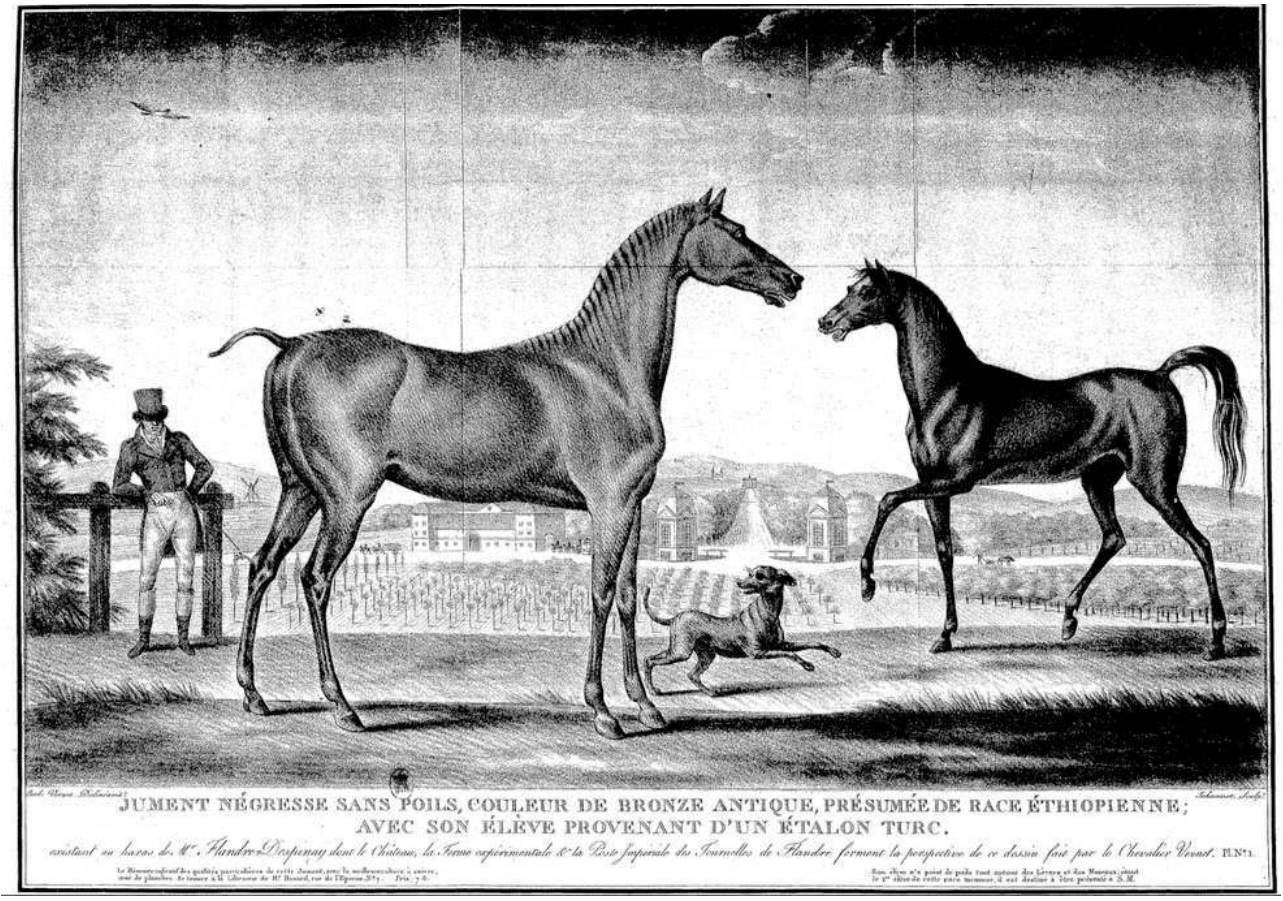

Jument présumée de race éthiopienne avec son produit issu d'un étalon du dépôt d'étalons de Cluny. Gravure d'après Carle Vernet contenue dans FLANDRE D'ESPINAY. De l'influence du grand propriétaire sur la prospérité agricole et commerciale, lorsqu'il s'occupe de haras d'expériences et qu'il établit des fermes expérimentales. Paris : Mme Huzard, 1809.

Repro. Bibliothèque nationale de France. (c) Bibliothèque nationale de France.

Dans la circonscription de Cluny, deux races en particulier retiennent l'intérêt.

Dans les départements que je viens de citer, celui de Saône-et-Loire semble tenir le premier rang. Il se compose en partie du Charolais et d'une portion du Morvan. Les chevaux de ces deux cantons ont acquis depuis longtemps à raison de leurs qualités une réputation bien méritée ${ }^{10}$.

Le fait que ces races aient été des entités bien reconnues, qui plus est jouissant d'une certaine notoriété dépassant les terres bourguignonnes, légitime l'envie d'approfondir la connaissance que nous en avons. Surtout, leur effectif autorise une étude plus fouillée. Les moyens pour y parvenir peuvent sembler dérisoires, mais il existe malgré tout un critère aisément observable et quantifiable : la taille. Il s'agit d'une donnée insuffisante et insatisfaisante qui a néanmoins le mérite d'être objective et qui permet, sinon d'avoir une idée fidèle de l'apparence du cheval, du moins d'en « évaluer les potentialités techniques d'utilisation par l'homme $»^{11}$. L'augmentation de la taille étant systématiquement recherchée et considérée comme un progrès, l'observation de son évolution contribue à donner une idée de l'influence du dépôt d'étalons (et ce, même si la génétique n'est pas le 
seul facteur d'amélioration mais partage au contraire la responsabilité d'un tel succès avec le perfectionnement des conditions agronomiques). Les registres de monte offrent une série exploitable pour ces deux races fameuses, ainsi que pour les races comtoise, normande et bressane (fig. 9). Il résulte de leur étude qu'en une quinzaine d'années toutes ces races ont crû, certaines de manière presque insensible $(0,4 \mathrm{~cm}$ de croissance pour les juments normandes, $0,5 \mathrm{~cm}$ pour la race bressane), d'autres d'une manière beaucoup plus perceptible : la race morvandelle - qui était certes celle dont la taille de départ était la plus réduite et par conséquent la plus sujette à progression - connut dans ce très court laps de temps une augmentation de près de 3 centimètres.

Figure 9

\begin{tabular}{c|c|c|c|c|c}
\hline \multirow{2}{*}{ race } & \multicolumn{2}{|c|}{$1807-1808-1809$} & \multicolumn{2}{c|}{1822} & \multirow{2}{*}{ croissance $(\mathrm{cm})$} \\
\cline { 2 - 6 } & taille moyenne $(\mathrm{m})$ & effectif & taille moyenne $(\mathrm{m})$ & effectif & 1 \\
\hline charolaise & $\mathbf{1 , 4 5 1}$ & 323 & $\mathbf{1 , 4 6 1}$ & 74 & 1,2 \\
\hline comtoise & 1,473 & 92 & 1,485 & 97 & 0.5 \\
\hline bressane & 1,477 & 109 & 1,482 & 106 & 0.4 \\
\hline normande & 1,508 & 88 & 1,512 & 103 & 2.8 \\
\hline morvandelle & 1,445 & 91 & 1,473 & \\
\hline
\end{tabular}

Tableau évolutif et comparatif de la taille moyenne de différentes races de juments dans la circonscription du dépôt d'étalons de Cluny (1807-1822).

Repro. Souvré, Amandine. (c) Amandine Souvré.

\section{Le recours à la notion de type}

Ainsi, l'absence de définition objective n'empêche pas la notion de race d'avoir une réelle acuité au début du XIX ${ }^{e}$ siècle. Par conséquent, et en dépit d'une inévitable imprécision, l'historien ne doit pas renoncer à la prendre en compte. L'on aurait tort cependant de lui prêter une importance pouvant confiner à l'anachronisme : si la notion de race était bel et bien présente dans les esprits, certains faits nous amènent à nuancer sa portée. Au nombre de ces réserves figure la part non négligeable d'étalons issus de croisement dans l'effectif des reproducteurs du dépôt : 40,5\% d'entre eux comptaient deux qualificatifs de race ou plus dans leurs ascendants. Le métissage pouvait aller jusqu'à l'extrême, comme l'atteste le cas du cheval baptisé « Le Lyon ", entré à Cluny en 1812 avec du sang espagnol, napolitain, normand et dombiste. On imagine aisément combien il était illusoire, dans ces conditions, d'avoir une ligne d'élevage cohérente et de produire " dans une race ». La race pouvait même s'avérer très secondaire : certaines juments amenées plusieurs fois à la saillie (la règle voulait que l'on ait droit à trois sauts) étaient parfois indifféremment livrées à tel ou tel étalon, de sorte que l'on aurait été bien en peine d'affirmer avec certitude la race du produit.

Il est d'autant plus nécessaire de ne pas se cantonner à cette notion qu'elle peut être parfois supplantée par une autre, plus pertinente. Ainsi, la désignation de "normand " englobait manifestement des réalités très différentes : le dépôt de Cluny en accueillit qui furent aussi bien qualifiés de "selle » que de "carrossier » et même de " trait », emplois spécifiques appelant pourtant des physiques fort distincts! Les divers usages du cheval offrent un autre moyen que la race de caractériser les équidés. Et l'on doit se demander si la taxinomie sur laquelle ils débouchent n'avait pas davantage de sens pour les contemporains. C'est en tous cas moins de la race que du «type » dont se préoccupaient les inspecteurs généraux des Haras quand il leur fallait estimer les besoins pour la remonte de l'établissement. L'enjeu majeur de cette question et les tensions à propos de 
l'inadéquation des étalons concernaient d'ailleurs le type des chevaux à produire et non leur race : les éleveurs, même en dehors des zones où un tel élevage était traditionnel, même avec une poulinière de selle, aspiraient majoritairement à faire naître des chevaux de trait alors que l'administration, sans l'avouer, faisait du cheval de selle - et plus précisément du cheval de cavalerie - un archétype et une priorité. La prédilection donnée au type sur la race est encore très sensible dans les écrits de certains inspecteurs généraux qui ne cherchaient pas à décrire et caractériser les populations de juments des différentes contrées autrement qu'en précisant l'arme à laquelle elles pourraient fournir des poulains - étant entendu que les qualificatifs d'arme (chasseurs, hussards, cavalerie lourde, artillerie, etc.) constituent un prolongement de la catégorisation tripartite « selle, carrossier, trait ». De la même façon, c'est en fonction de ces trois classes fonctionnelles que la circulaire ministérielle de 1812 exigeait le dénombrement des chevaux présents sur le territoire.

Race et type, toutefois, ne sont pas deux concepts à opposer. Le premier est une qualité intrinsèque, le second dépend d'un facteur externe, lié aux différentes utilisations du cheval par l'homme. Mais tous deux ont forgé un langage en même temps qu'ils modelaient des physiques différenciés. Seulement, les divers usages ont opéré ces infléchissements depuis une période bien antérieure à celle où l'on s'est occupé de classer, d'améliorer, de diversifier ou au contraire de restreindre les races. C'est pourquoi le type est sans doute, pour le moment, à considérer comme l'expression la plus flagrante de l'influence humaine sur le cheval et la preuve que c'est bien par le travail que l'on a cherché, à l'origine, à le définir.

\section{BIBLIOGRAPHIE}

BOUGLER, Jacques. «La race et les livres généalogiques ». Dans Le concept de race en zootechnie. Paris : Société d'ethnozootechnie, 1982.

DENIS, Bernard. «Les races de chevaux en France au XVIII ${ }^{\mathrm{e}}$ siècle et les idées relatives à leur amélioration ». In Situ, 2012, $\mathrm{n}^{\circ} 18$.

PELLEGRINI, Patricia. « De l'idée de race animale et de son évolution dans le milieu de l'élevage ». Ruralia, 1999, t. 5.

\section{NOTES}

1. - Voir les sites : http://www.sudoc.abes.fr//DB=2.1/SET=2/TTL=44/CMD? ACT=SRCHA\&IKT=1016\&SRT=RLV\&TRM=amandine+souvr\%C3\%A9. Et http:// theses.enc.sorbonne.fr/2014/souvre [consultés 15/09/2015).

2. - PELLEGRINI, Patricia. "De l'idée de race animale et de son évolution dans le milieu de l'élevage ». Ruralia, 1999, t. 5.

3. - Le registre-matricule français entend par "race pure» les chevaux de pur sang arabes, barbes, turcs et persans. Il s'avère toutefois que les chevaux de pur sang anglais n'en ont pas été 
exclus : de fait il existe dans le premier volume, publié en 1838 seulement, une section orientale et une section anglaise. BLOMAC, Nicole de. La Gloire et le jeu : des hommes et des chevaux, 1766-1866. Paris : Fayard, 1991, p. 195-197. BLOMAC, Nicole de et BOGROS, Denis. L'Arabe, premier cheval de sang. Paris : Crépin-Leblond, 1978, p. 158-159.

4. - LIZET, Bernadette. La bête noire : à la recherche du cheval parfait. Paris : Éditions de la Maison des sciences de l'homme, 1989.

5. - BOUGLER, Jacques. «La race et les livres généalogiques ». Dans Le concept de race en zootechnie. Paris : Société d'ethnozootechnie, 1982, p. 72.

6. - ROCHE, Daniel. « Les chevaux de la République : l'enquête de l'an III ». Revue d'histoire moderne et contemporaine, 2008 , t. 55-4, p. 83.

7. - Arch. nat., $\mathrm{F}^{10} 738$, rapport de l'inspecteur général Lastic, 1819.

8. - Ibid., rapport de l'inspecteur général Ligniville, 5 avril 1810.

9. - Voir le développement consacré à ce sujet dans DENIS, Bernard. «Les races de chevaux en France au XVIII ${ }^{\mathrm{e}}$ siècle et les idées relatives à leur amélioration ». In Situ, 2012, $\mathrm{n}^{\circ} 18$. Voir le site : http://insitu.revues.org/9677 [document électronique]. Paris : Ministère de la Culture et de la Communication. Arch. dép. Saône-et-Loire, 4 ETP 397, 4 ETP 398, 4 ETP 460-463.

10. - Arch. nat, $\mathrm{F}^{10} 738$, rapport de l'inspecteur général Lastic, 14 octobre 1817.

11. - BLOMAC, Nicole de. «Le cheval de guerre entre le dire et le faire ». Dans ROCHE, Daniel. Le cheval et la guerre: $d u X^{e}$ au XXe siècle. Versailles: Association pour l'Académie d'art équestre de Versailles, 2002, p. 57.

\section{RÉSUMÉS}

Fruit d'une lente maturation, la notion de «race» oppose de nombreuses résistances à l'historien. Désormais indispensable à l'identification des équidés, elle était, au début du XIX siècle encore, une idée aux contours diffus qu'il est toutefois possible de mieux circonscrire grâce à une étude du cheptel. Elle s'efface devant le «type ", qui permet de dépasser cette qualité intrinsèque en définissant le cheval par sa fonction au travail et qui exprime, par le langage et les physiques qu'il forge, l'expression la plus flagrante de l'influence humaine sur le cheval.

Horse categories: race and type. The slowly evolving notion of 'race' is one that creates many obstacles for the historian. It is indispensable today for the identification of equine animals, but at the beginning of the nineteenth century it was still only a vague notion, which is nevertheless possible appreciate through a study of livestock. It is superseded by the 'type', which allows us to go beyond inherent qualities by defining the horse in terms of its work function. Through the language and the morphologies it shapes, this expresses the most flagrant expression of the human influence over the horse.

\section{INDEX}

Mots-clés : histoire, élevage, race, Saône-et-Loire, XIXe siècle, Premier Empire, cheval, studbook

Keywords : history, breeding, race, Saône-et-Loire, nineteenth century, First Empire, stud book 
AUTEUR

AMANDINE SOUVRÉ

archiviste paléographe amandine.souvre@hotmail.fr 